This article is licensed under the Creative Commons Attribution-NonCommercial 4.0 International License (CC BY-NC) (http://www.karger.com/Services/OpenAccessLicense) Usage and distribution for commercial purposes requires written permission.

\title{
Genomic Profiling of Two Histologically Distinct Rare Urothelial Cancers in a Clinical Setting to Identify Potential Therapeutic Options for Treatment and Management of Disease
}

\author{
Andrew N. Hesse ${ }^{a}$ William Fabricius ${ }^{b}$ Christian A. Thomas ${ }^{c}$ \\ Ramesh Gaindh $^{d}$ Robert Christman ${ }^{\mathrm{e}}$ Pavalan Selvam $^{\mathrm{a}}$ \\ Matthew Prego ${ }^{a} \quad$ Gregory Lewis $^{a}$ Jasmina Uvalic ${ }^{a}$ Daniel Bergeron ${ }^{a}$ \\ Shelbi Burns $^{a} \quad$ Bridgette Sisson $^{a}$ Kevin Kelly $^{a}$ Jens Rueter ${ }^{f}$ \\ Honey V. Reddi ${ }^{\mathrm{a}}$ \\ aThe Jackson Laboratory for Genomic Medicine, Farmington, CT, USA; ${ }^{\text {bPen Bay Medical }}$ \\ Center, Rockport, ME, USA; 'New England Cancer Specialists, Scarborough, ME, USA; \\ dMid Coast Hospital, Brunswick, ME, USA; eDepartment of Pathology, Maine Medical \\ Center, Portland, ME, USA; The Maine Cancer Genomics Initiative, The Jackson \\ Laboratory, Augusta, ME, USA
}

A.N. Hesse, W. Fabricius, C.A. Thomas, R. Gaindh, and R. Christman contributed equally to this work. J. Rueter and H.V. Reddi: equal contribution. 


\section{Case Reports in Oncology}

Case Rep Oncol 2018;11:196-205

DOI: $10.1159 / 000487882$

(c)

C 2018 The Author(s). Published by S. Karger AG, Base www.karger.com/cro

Hesse et al.: Genomic Profiling of Rare Urothelial Cancers

\title{
Keywords
}

Genomic profiling · Next-generation sequencing · Urothelial cancer

\begin{abstract}
Molecular profiling of urothelial cancers for therapeutic and prognostic potential has been very limited due to the absence of cancer-specific targeted therapies. We describe here 2 clinical cases with a histological diagnosis of an invasive sarcomatoid and a poorly differentiated carcinoma favoring urothelial with some neuroendocrine differentiation, two of the rarer types of urothelial cancers, which were evaluated for mutations in 212 genes for singlenucleotide variants and copy-number variants and 53 genes for fusions associated with solid tumors. In both cases, we identified variants in 2 genes, ARID1A and CDKN2A, indicative of the role of dysregulation of chromatin remodeling and cell cycle control as being common features of bladder cancer, consistent with the proposed model of tumorigenesis in these rare, highly aggressive pathological subtypes. The presence of a KRAS mutation in the poorly differentiated cancer and a TP53 mutation in the sarcomatoid tumor is indicative of a distinctive profile and adds a potential layer of molecular stratification to these rarer histological subtypes. We present a comparative analysis of the histological, clinical, and molecular profile of both cases and discuss the potential to delineate these tumors at the molecular level keeping in mind the possible therapeutic implications.

(C) 2018 The Author(s)

Published by S. Karger AG, Basel
\end{abstract}

\section{Introduction}

Bladder cancer is the most common malignancy involving the urinary system and the ninth most common malignancy worldwide [1], responsible for $\sim 150,000$ deaths per year worldwide and about 5\% of all new cancers in the US [2]. Invasive urothelial carcinoma involving the urinary bladder is very well known for divergent differentiation [3], with transitional cell carcinoma being the most common histological type accounting for $90 \%$ of cases. Sarcomatoid carcinomas of the bladder are rare $(0.6 \%$ of bladder cancers - SEER Statistics) with only about 24 cases reported so far [4]. Similarly, neuroendocrine tumors of the bladder are equally rare ( $<0.5 \%$ of all bladder cancer malignancies) with $\sim 12$ cases being reported [5]. Prognosis with both variant morphologies is poor with an aggressive clinical course and rapid onset of metastatic disease. Given the rarity of the 2 variant morphotypes presented in the current analysis, it is difficult to draw any conclusions about the optimal treatment strategy or prognostic markers due to the small number of reported cases.

Here we present 2 cases (initial pathology, genomic findings, and clinical follow-up) of the rare pathological subtypes of urothelial cancer, a sarcomatoid tumor and a poorly differentiated urothelial carcinoma (PDUC) with some neuroendocrine differentiation. To our knowledge, this is the first report of these rare variant morphologies of urothelial carcinomas being described with clinical genomic profiling to identify potential targeted therapeutic approaches. 


\section{Case Reports in Oncology}

\section{Case Reports}

\section{PDUC with Some Neuroendocrine Differentiation}

A 70-year-old man with a diagnosis of invasive PDUC of the right lateral wall of the bladder was initially noted by his primary care provider to have persistent microscopic hematuria. However, cystoscopy conducted 2 months later did not identify any tumors. CT scans of the abdomen and pelvis with contrast were negative for metastatic disease. Three months later, he developed macroscopic hematuria and anemia and a new 3-4 cm broadbased nodular lesion was identified in the right lateral wall of the bladder on repeat cystoscopy. Transurethral resection of these lesions and several clots in the bladder were noted during that procedure and were removed, resulting in resolution of his gross hematuria. Pathology of these lesions noted a high-grade, muscle-invasive PDUC with some neuroendocrine differentiation of the right lateral wall of the bladder (Fig. 1a, b). He was clinically staged as stage II, cT2 cN0 cM0. A PET/CT scan done at the same time showed no evidence of regional or distant metastatic disease.

\section{Invasive Sarcomatoid Urothelial Carcinoma}

A 69-year-old man with a family history of pancreatic cancer in his mother and a brother initially underwent an evaluation for hematuria followed by a partial cystectomy when a cystoscopy identified a bladder diverticulum. Histological examination identified a highgrade bladder tumor with sarcomatoid features (Fig. 1c, d). Associated lymph nodes were negative for malignancy. The tumor had been resected with negative margins and no further treatment was advised at that time. After 9 months of surveillance, a repeat cystoscopy revealed the possibility of recurrent disease and restaging MRI scan of the abdomen performed at the same time revealed the presence of bulky pelvic and retroperitoneal adenopathy. A transurethral resection of a recurrent tumor in the trigone area showed invasive sarcomatoid urothelial carcinoma, which locally progressed as confirmed on CT scans 1 month later. Chemotherapy with gemcitabine and cisplatin was initiated, but after a brief period of clinical improvement, a repeat staging CT scan after about 2.5 months of treatment revealed progression of his pelvic and retroperitoneal disease. Given a high PD-L1 score of 95\%, treatment with atezolizumab was initiated but the patient progressed clinically after 2 cycles of treatment and then expired.

\section{Genomic Analyses and Clinical Interpretation}

Ultrasound-guided core-needle biopsies were histologically evaluated for diagnosis of malignancy (Fig. 2) and formalin-fixed paraffin-embedded (FFPE) samples were shipped to The Jackson Laboratory for Genomic Medicine (JAX) CLIA lab by the ordering physicians (W.F. and C.A.T.) to be processed using a clinically validated (CLIA/CAP) assay called the ActionSeq Plus ${ }^{\mathrm{TM}}$. The assay included a DNA-based panel comprising 212 cancer-related genes for which all coding exons are sequenced (to allow for detection of somatic singlenucleotide variants, indels, and copy-number variants) and a RNA-based panel for 53 genes known to form fusions. Data were analyzed to identify variants of actionability, i.e., those with therapeutic, prognostic, or diagnostic relevance. 


\section{Case Reports in Oncology}

Case Rep Oncol 2018;11:196-205

DOI: $10.1159 / 000487882$

Hesse et al.: Genomic Profiling of Rare Urothelial Cancers

Two genes were mutated in both cases; actionable variants were identified in ARID1A (p.Q1172* - PDUC and p. H782Tfs*51 - sarcomatoid) and CDKN2A (p. N42Kfs*77 - PDUC and p. W110* - sarcomatoid). Additionally, we identified an actionable KRAS (p.G12D) variant in the PDUC and 2 actionable variants in the tumor suppressors TP53 (p. R273C) and FBXW7 (p. R465C) in the sarcomatoid cancer. While no variants of uncertain significance were identified in the PDUC, we did identify 1 variant of uncertain significance in NFE2L2 (p.E82V) in the sarcomatoid tumor (Table 1). No actionable copy-number variants or fusions were identified in either case. A comprehensive summary of the potential therapies, variant classification, FDA-approved therapies and potential clinical trials identified for the patientspecific mutational profile is listed in Table 2. While there were no FDA-approved targeted therapies identified for urothelial tumors in any of the relevant drug classes, FDA-approved drugs for these mutations in other cancers (metastatic or locally advanced solid tumors) were listed in the genomic testing report as potential options for off-label use, consistent with the AMP/ASCO guidelines for reporting of somatic variants [6].

\section{Treatment Outcomes}

\section{PDUC with Some Neuroendocrine Differentiation}

The patient was initially started on neoadjuvant chemotherapy with cisplatin-etoposide (i.v. cisplatin $80 \mathrm{mg} / \mathrm{m}^{2}$ on day 1, i.v. etoposide $80 \mathrm{mg} / \mathrm{m}^{2}$ on days 1,2 , and 3). This was planned for 4 cycles. During the completion of this first cycle, a revision of his pathology stated that the neuroendocrine component of his tumor was minimal, with scant evidence of neuroendocrine cells and very weak stain synaptophysin, thus favoring the diagnosis of PDUC. After a multidisciplinary discussion, the patient's neoadjuvant chemotherapy regimen was changed to dose-dense methotrexate-vinblastin-doxorubicin-cisplatin (ddMVAC) (i.v. cisplatin $70 \mathrm{mg} / \mathrm{m}^{2}$, i.v. methotrexate $30 \mathrm{mg} / \mathrm{m}^{2}$, i.v. vinblastin $3 \mathrm{mg} / \mathrm{m}^{2}$, I.V. doxorubicin 30 $\mathrm{mg} / \mathrm{m}^{2}$ on day 1 every 2 weeks), a category $1 \mathrm{NCCN}$ guideline recommendation.

The patient completed 3 cycles of neoadjuvant ddMVAC without serious complications except for moderate cytopenia, fatigue, poor appetite, and nausea. Two weeks after completion of that regimen, restaging CT scans of the abdomen, pelvis, and chest showed no radiological evidence of disease. The plan is now to perform a radical cystectomy, to be followed likely by active monitoring for development of metastatic disease. There remains a significant risk for metastatic disease recurrence and having the next-generation sequencing test results may help identify future treatment options or clinical trials if the patient develops metastatic disease.

\section{Sarcomatoid Urothelial Carcinoma}

The patient passed away in the interim during genomic testing and was unable to benefit from the results of genomic testing. 


\section{Case Reports in Oncology}

Case Rep Oncol 2018;11:196-205

DOI: $10.1159 / 000487882$

(C) 2018 The Author(s). Published by S. Karger AG, Basel www.karger.com/cro

Hesse et al.: Genomic Profiling of Rare Urothelial Cancers

\section{Discussion}

Precision medicine aims to identify novel targets for treating rare and difficult-to-treat cancers. To advance the field and identify novel treatment options for patients, broad molecular profiling is needed. Here we describe the molecular profiling results of 2 cases with urothelial cancers that identified potential treatment options for the patients. We identified some interesting profiles which offer the opportunity to identify new treatment options and may support novel molecular disease classifications in the future.

Mutations in 2 genes, ARID1A and CDKN2A, were identified in both our cases and they have been shown to occur in 25 and $5 \%$ of urothelial carcinomas, respectively, indicative of the role of dysregulation of chromatin remodeling and cell cycle control in these rare, highly aggressive pathological subtypes. Evaluation of 347 cases across a broad complement of cancers demonstrated that $C D K N 2 A$ was more likely to be independently associated with ARID1A in a multivariate analysis (OR D 3.98, 95\% CI: 1.36-11.8, $p$ D 0.01, $p=0.01$ ).

In addition, we identified a mutation in KRAS in 1 of the 2 cases (PDUC). Mutations in the RAS oncogenes (HRAS, KRAS, and NRAS) are found in 11-13\% of bladder tumors and occur in all stages and grades, with mutations in KRAS being the least frequent [7]. Profiling of urothelial cancers across multiple studies have not identified co-occurrence of KRAS with CDKN2A mutations [8], however, a single study has reported the co-occurrence of a KRAS substitution with an ARID1A truncation mutation in a stage IV high-grade urothelial carcinoma in a 51-year-old male [9] indicative of the rarity of this genomic profile. The cooccurrence of the ARID1A and KRAS mutation in the PDUC could therefore possibly account for the aggressive and poorly differentiated nature of the tumor. In contrast to the PDUC, the sarcomatoid urothelial carcinoma had actionable mutations in 3 other genes (TP53, FBXW7, and NFE2L2) known to harbor aberration in bladder cancer. TP53 is the most commonly mutated gene in bladder cancer observed at a frequency of $\sim 15 \%$ in non-muscle-invasive tumors as is the sarcomatoid tumor. Mutations in TP53 have been shown to co-occur with either ARID1A or CDKN2A [9] with aberration in the CDK pathway genes being the second most common mutation across most cancers next to TP53 [10]. Mutations in FBXW7 are observed in $10 \%$ of bladder cancers across all stages [7] and mutations in NFE2L2 are observed in $\sim 8-11 \%$ of bladder tumors [11], particularly the basal subtype [12], based on samples in TCGA suggesting a potential role for the oxidative stress pathway in progression of bladder cancer. While co-occurrence of FBXW7 mutations with TP53 has been reported in a single case report, of a high-grade, advanced-stage tumor from a 71-year-old woman, from the lymph node metastasis specimen which had a micropapillary architecture [9], mutations in NFE2 L2 have not been shown to co-occur with TP53 mutations [12] across a comprehensive study evaluating $\sim 400$ bladder cancers, contributing to the novelty of this genomic profile and providing further insight into the possible genomic changes involved in the development and evolution of sarcomatoid urothelial carcinoma.

There are currently no molecularly targeted agents approved for the treatment of bladder cancer despite a significant amount of effort in recent times to profile bladder tumors. By following the AMP/CAP/ASCO guidelines on reporting actionability of cancer-specific genes, we were able to identify potential drug classes for clinical consideration (Table 2). These included FDA-approved drugs (for a different indication) as well as some experimental therapies. In addition, we were able to identify a number of clinical trials for each one of the pa- 


\section{Case Reports in Oncology}

tients based on their genomic profile, although all of the trials were recruiting patients broadly across solid tumors. Most noteworthy is a trial listed for the ARID1A and KRAS mutations (NCT02576444) which is a phase II study of the PARP inhibitor olaparib (AZD2281) alone and in combination with AZD1775, AZD5363, or AZD2014 in advanced solid tumors across different molecular profiles including any mutation in ARID1A, as well as protocoldefined mutations in KRAS and TP53. The TP53 mutation identified in the sarcomatoid case did not fit inclusion criteria, but the mutations in ARID1A and KRAS were eligible, resulting in the inclusion of this trial in the genomic testing report. All in all, molecular profiling has identified a number of genomic-marker-driven treatment approaches for patients with urothelial cancers, but these treatments should be accessed through clinical trial enrollment rather than off-label use of drugs.

Though the ARID1A and CDKN2A genes identified in our cases are amongst those known to be commonly associated with urothelial carcinoma, to our knowledge this study is the first clinical case report comparing and identifying unique genomic profiles in 2 rare pathological subtypes of urothelial cancer. The identification of the co-occurring combinations of ARID1A/KRAS in the PDUC and the TP53/FBXW7 in the sarcomatoid subtype adds a layer of potential stratification across these two histological subtypes. Further, our documentation of these rare co-occurrences in high-grade urothelial carcinomas adds an additional case report to the existing literature of single cases currently reported for each combination, amplifying the potential of broad-based targeted clinical trials. In addition, given the rarity of these tumors, with only 24 clinical cases of sarcomatoid tumors [4] and $\sim 12$ cases of urothelial carcinoma with neuroendocrine component [5] being reported, our evaluation also adds to the knowledgebase of these rare urothelial carcinoma morphologies, both from a genomic profile perspective as well as identification of potential targeted therapies and trials since there are currently no targeted FDA-approved therapies specific for urothelial cancer.

\section{Acknowledgment}

We gratefully acknowledge the participation of the patients. The authors are grateful for the funding support from the Harold Alfond ${ }^{\circledR}$ Foundation that facilitated the genomic testing in collaboration with The Jackson Laboratory as part of the Maine Cancer Genomics Initiative (MCGI) led by J.R.

\section{Statement of Ethics}

Patient specimens were sent to the CLIA/CAP-accredited laboratory at The Jackson Laboratory for Genomic Medicine as part of the patients' routine care and management for genomic testing. Informed written consent was obtained by the ordering physician from the patient for tumor profiling using the JAX ActionSeq Plus ${ }^{\mathrm{TM}}$ assay. The use of data sets is allowed for research reports and scientific publications. 


\section{Disclosure Statement}

The authors declare that there are no conflicts of interest.

\section{References}

1 Ploeg M, Aben KK, Kiemeney LA. The present and future burden of urinary bladder cancer in the world. World J Urol. 2009 Jun;27(3):289-93.

2 Andreassen BK, Aagnes B, Gislefoss R, Andreassen M, Wahlqvist R. Incidence and Survival of urothelial carcinoma of the urinary bladder in Norway 1981-2014. BMC Cancer. 2016 Oct;16(1):799.

3 Humphrey PA, Moch H, Cubilla AL, Ulbright TM, Reuter VE. The 2016 WHO Classification of Tumours of the Urinary System and Male Genital Organs-Part B: Prostate and Bladder Tumours. Eur Urol. 2016 Jul;70(1):106-19.

4 Lu W, Wang Y, Li Y, Cao Y, Han H, Zhou F. Sarcomatoid urothelial carcinoma with chondrosarcomatous differentiation of the ureter: A case report and review of the literature. Oncol Lett. 2017 Mar;13(3):1331-7.

5 Amin MB. Histological variants of urothelial carcinoma: diagnostic, therapeutic and prognostic implications. Mod Pathol. 2009 Jun;22(S2 Suppl 2):S96-118.

6 Li MM, Datto M, Duncavage EJ, Kulkarni S, Lindeman NI, Roy S et al. Standards and Guidelines for the Interpretation and Reporting of Sequence Variants in Cancer: A Joint Consensus Recommendation of the Association for Molecular Pathology, American Society of Clinical Oncology, and College of American Pathologists. J Mol Diagn. 2017 Jan;19(1):4-23.

7 Kompier LC, Lurkin I, van der Aa MN, van Rhijn BW, van der Kwast TH, Zwarthoff EC. FGFR3, HRAS, KRAS, NRAS and PIK3CA mutations in bladder cancer and their potential as biomarkers for surveillance and therapy. PLoS One. 2010 Nov;5(11):e13821.

8 Grivas P, Zargar H, Ercole CE, Kovac E, Remer EM, Karim W et al. Assessment of sarcopenia as predictor of response and outcome after neoadjuvant chemotherapy (NAC) and radical cystectomy (RC) in muscleinvasive bladder cancer (MIBC). J Clin Oncol. 2015;33(15_suppl):e15512.

9 Ross JS, Wang K, Al-Rohil RN, Nazeer T, Sheehan CE, Otto GA et al. Advanced urothelial carcinoma: nextgeneration sequencing reveals diverse genomic alterations and targets of therapy. Mod Pathol. 2014 Feb;27(2):271-80.

10 Kato S, Schwaederle M, Daniels GA, Piccioni D, Kesari S, Bazhenova L et al. Cyclin-dependent kinase pathway aberrations in diverse malignancies: clinical and molecular characteristics. Cell Cycle. 2015;14(8):1252-9.

11 Real FX, Boutros PC, Malats N. Next-generation sequencing of urologic cancers: next is now. Eur Urol. 2014 Jul;66(1):4-7.

12 Kim J, Akbani R, Creighton CJ, Lerner SP, Weinstein JN, Getz G et al. Invasive Bladder Cancer: Genomic Insights and Therapeutic Promise. Clin Cancer Res. 2015 Oct;21(20):4514-24. 


\section{Case Reports in Oncology}

Case Rep Oncol 2018;11:196-205

DOI: $10.1159 / 000487882$

(c) 2018 The Author(s). Published by S. Karger AG, Basel www.karger.com/cro

Hesse et al.: Genomic Profiling of Rare Urothelial Cancers

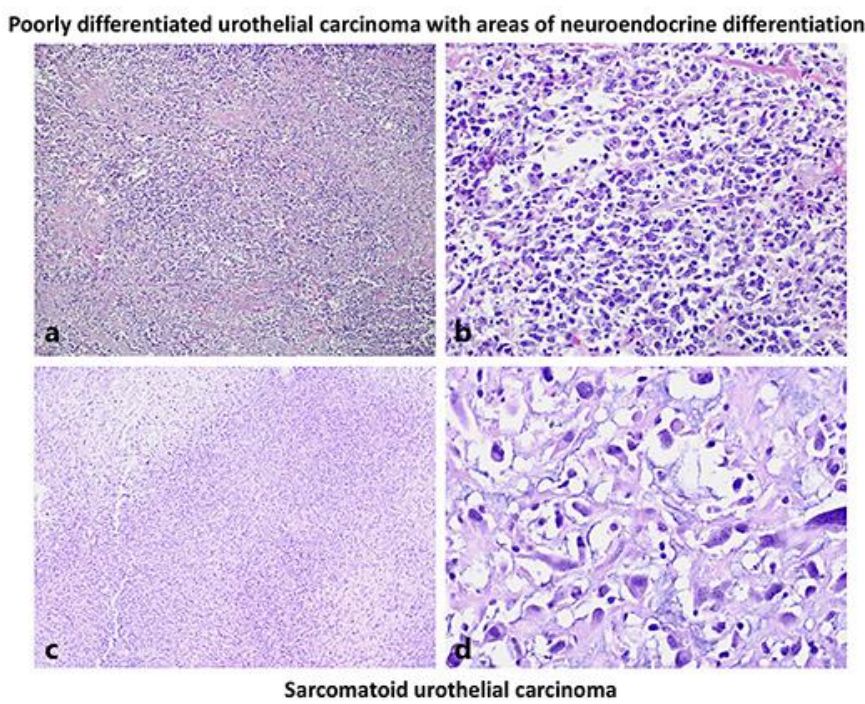

Fig. 1. a, b Pathology sections of a poorly differentiated urothelial carcinoma: $\times 4$ (a), $\times 40$ (b). c, d Pathology sections of a sarcomatoid carcinoma: $\times 20$ (c), $\times 40$ (d).

\section{Poorly differentiated urothelial carcinoma with areas of neuroendocrine differentiation}

\section{Sarcomatoid urothelial carcinoma}
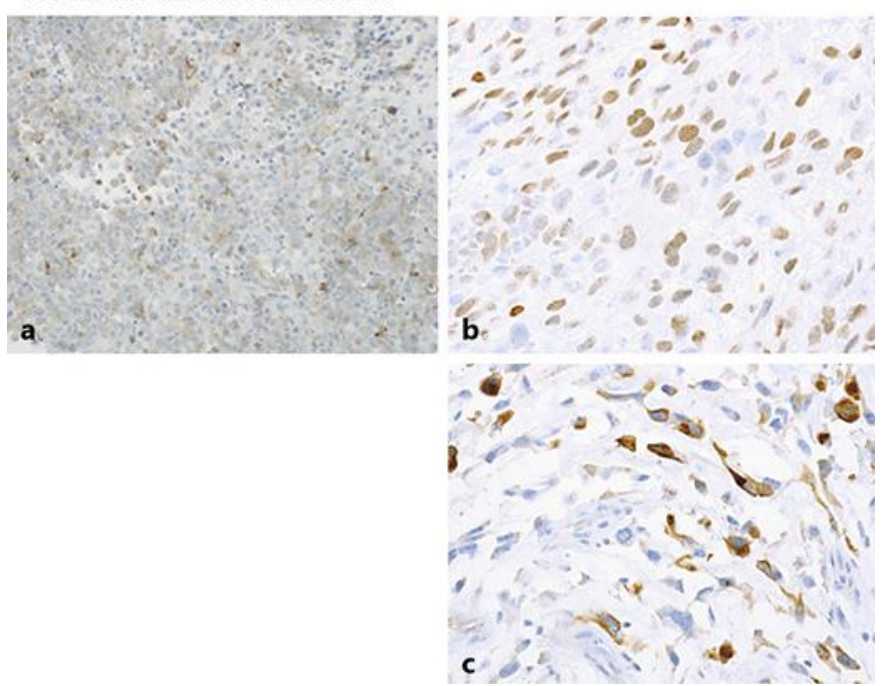

Fig. 2. Immunostained sections of the tumors: synaptophysin (poorly differentiated urothelial carcinoma) (a), GATA3 (sarcomatoid) (b), and CAM5.2 (sarcomatoid) (c). 


\section{Case Reports in Oncology}

\begin{tabular}{l|l}
\hline Case Rep Oncol 2018;11:196-205 \\
\hline DOI: 10.1159/000487882 & $\begin{array}{l}\text { (c) 2018 The Author(s). Published by S. Karger AG, Basel } \\
\text { www.karger.com/cro }\end{array}$ \\
\hline
\end{tabular}

Hesse et al.: Genomic Profiling of Rare Urothelial Cancers

Table 1. Variants reported in both cases

\begin{tabular}{|c|c|c|c|c|c|c|c|}
\hline Histology & Gene & $\begin{array}{l}\text { Protein } \\
\text { change }\end{array}$ & cDNA & Transcript & Coordinate & $\begin{array}{l}\text { Variant } \\
\text { type }\end{array}$ & $\begin{array}{l}\text { Predicted } \\
\text { effect }\end{array}$ \\
\hline \multicolumn{8}{|l|}{ Case 1} \\
\hline \multirow{3}{*}{$\begin{array}{l}\text { PDUC with areas } \\
\text { of neuroendocrine } \\
\text { differentiation }\end{array}$} & ARID1A & p.Q1172* & c. $3514 \mathrm{C}>\mathrm{T}$ & NM_006015.4 & chr1:26772607 & Transition & Missense \\
\hline & CDKN2A & p.N42Kfs77* & c.126_127delTA & NM_000077.4 & chr9:21974700 & Deletion & FS/truncation \\
\hline & KRAS & p.G12D & c. $35 \mathrm{G}>\mathrm{A}$ & NM_033360.3 & chr12:25245350 & Transition & Missense \\
\hline \multicolumn{8}{|l|}{ Case 2} \\
\hline \multirow{4}{*}{$\begin{array}{l}\text { Sarcomatoid } \\
\text { urothelial } \\
\text { carcinoma }\end{array}$} & ARID1A & p.H782Tfs*51 & c.2343delA & NM_006015.4 & chr1:26762242 & Deletion & FS/truncation \\
\hline & CDKN2A & p.W110* & c. $330 \mathrm{G}>\mathrm{A}$ & NM_000077.4 & chr9:21971029 & Transition & Nonsense \\
\hline & FBXW7 & p.R465C & c. $1393 \mathrm{C}>\mathrm{T}$ & NM_033632.3 & chr4:152328233 & Transition & Missense \\
\hline & TP53 & p.R273C & c. $817 \mathrm{C}>\mathrm{T}$ & NM_000546.5 & chr17:7673803 & Transition & Missense \\
\hline
\end{tabular}

PDUC, poorly differentiated urothelial carcinoma; FS, frameshift mutation. 
Table 2. Clinically significant variants with potential therapies and clinical trials listed in genomic testing reports

\begin{tabular}{|c|c|c|c|c|c|c|}
\hline Histology & Gene & Classification & Drug classes & $\begin{array}{l}\text { FDA approved } \\
\text { for tumor type }\end{array}$ & $\begin{array}{l}\text { FDA approved for } \\
\text { other indications }\end{array}$ & $\begin{array}{l}\text { Potential } \\
\text { clinical trials }\end{array}$ \\
\hline \multicolumn{7}{|l|}{ Case 1} \\
\hline \multirow[t]{7}{*}{$\begin{array}{l}\text { PDUC with areas } \\
\text { of neuroendocrine } \\
\text { differentiation }\end{array}$} & \multirow[t]{2}{*}{$\begin{array}{l}\text { ARID1A } \\
\text { c.3514C >T }\end{array}$} & \multirow[t]{2}{*}{ Tier II } & $\sqrt{ }$ PARP inhibitor & None & \multirow{2}{*}{$\begin{array}{l}\text { Olaparib }^{1} \\
\text { Niraparib }^{2} \\
\text { Rucaparib }^{2} \\
\text { N/A }\end{array}$} & NCT02576444 \\
\hline & & & O AKT inhibitor & N/A & & NCT02576444 \\
\hline & \multirow[t]{2}{*}{$\begin{array}{l}\text { CDKN2A } \\
\text { c.126_127delTA }\end{array}$} & \multirow[t]{2}{*}{ Tier II } & $\sqrt{\text { CDK4/6 inhibitor }}$ & None & \multirow{2}{*}{$\begin{array}{l}\text { Palbociclib } 1 \\
\text { Ribociclib }^{2} \\
\text { Abemaciclib }^{2} \\
\text { N/A }\end{array}$} & $\begin{array}{l}\text { NCT02693535, } \\
\text { NCT02334527 }\end{array}$ \\
\hline & & & O Multikinase inhibitor & N/A & & $\begin{array}{l}\text { NCT02478320, } \\
\text { NCT02540876 }\end{array}$ \\
\hline & \multirow[t]{3}{*}{$\begin{array}{l}\text { KRAS } \\
\text { c.35G }>\text { A }\end{array}$} & \multirow[t]{3}{*}{ Tier II } & $\sqrt{\text { MEK inhibitor }}$ & None & $\begin{array}{l}\text { Cobimetinib } \\
\text { Trametinib }\end{array}$ & $\begin{array}{l}\text { NCT01827384, } \\
\text { NCT02022982 } \\
\text { NCT03108131, } \\
\text { NCT02079740 } \\
\text { NCT03162627 }\end{array}$ \\
\hline & & & O ERK inhibitor & N/A & $\mathrm{N} / \mathrm{A}$ & $\begin{array}{l}\text { NCT02857270, } \\
\text { NCT03051035 }\end{array}$ \\
\hline & & & O KRAS ASO inhibitor & N/A & N/A & NCT03101839 \\
\hline \multicolumn{7}{|l|}{ Case 2} \\
\hline \multirow{9}{*}{$\begin{array}{l}\text { Sarcomatoid } \\
\text { urothelial } \\
\text { carcinoma }\end{array}$} & \multirow[t]{3}{*}{$\begin{array}{l}\text { ARID1A } \\
\text { c.2343delA }\end{array}$} & \multirow[t]{3}{*}{ Tier II } & $\sqrt{ }$ PARP inhibitor & None & \multirow{3}{*}{$\begin{array}{l}\text { Olaparib }^{1} \\
\text { Niraparib }^{2} \\
\text { Rucaparib }^{2} \\
\text { N/A }\end{array}$} & NCT02576444 \\
\hline & & & & & & \\
\hline & & & O AKT inhibitor & N/A & & NCT02576444 \\
\hline & \multirow[t]{2}{*}{$\begin{array}{l}\mathrm{CDKN} 2 \mathrm{~A} \\
\text { c. } 330 \mathrm{G}>\mathrm{A}\end{array}$} & \multirow[t]{2}{*}{ Tier II } & $\sqrt{\text { CDK4/6 inhibitor }}$ & None & \multirow{2}{*}{$\begin{array}{l}\text { Palbociclib }^{1} \\
\text { Ribociclib }^{2} \\
\text { Abemaciclib }^{2} \\
\text { N/A }\end{array}$} & $\begin{array}{l}\text { NCT02693535, } \\
\text { NCT02334527 }\end{array}$ \\
\hline & & & O Multikinase inhibitor & N/A & & $\begin{array}{l}\text { NCT02478320, } \\
\text { NCT02540876 } \\
\end{array}$ \\
\hline & \multirow{4}{*}{$\begin{array}{l}\text { FBXW7 } \\
\text { c.1393C }>\text { T } \\
\text { TP53 } \\
\text { c.817C }>\mathrm{T}\end{array}$} & Tier II & O CHK1 inhibitor & N/A & N/A & NCT02873975 \\
\hline & & \multirow[t]{3}{*}{ Tier II } & O P53 gene therapy & N/A & $\mathrm{N} / \mathrm{A}$ & NCT02842125 \\
\hline & & & O P53 vaccine & N/A & N/A & NCT02432963 \\
\hline & & & O WEE1 inhibitor & N/A & $\mathrm{N} / \mathrm{A}$ & NCT02576444 \\
\hline
\end{tabular}

PDUC, poorly differentiated urothelial carcinoma; $\sqrt{ }$, approved therapies; 0 , experimental therapies; Tier I, strong clinical significance; Tier II, potential clinical significance; Tier III, unknown clinical significance.

1 Currently being investigated in a clinical trial. 2 Additional "in-class" drug not currently under active investigation in a clinical trial. 